\title{
The Adaptive Social Hierarchy - A Self Organizing Network Based on Naturally Occurring Structures
}

\author{
Andrew Markham, Andrew Wilkinson \\ Department of Electrical Engineering \\ University of Cape Town \\ Rondebosch, 7701, South Africa \\ mrkand007@mail.uct.ac.za
}

\begin{abstract}
Wireless networks can be used for relaying information acquired by mobile animal borne tags. To date, no research has considered the large amount of diversity presented by the animal kingdom which impacts the design of the network. We consider here how the weight of the animal affects the size of the tag, and hence the energy that it can carry. We use a common structure in Nature, the social dominance hierarchy, and form a similar hierarchy based on energy. Nodes adjust their perceived rankings through continual tournaments using simple, locally applied rules to result in a stable and adaptive structure. We show that the number of levels in the hierarchy controls traffic density and consequently energy usage. To further conserve energy of low ranked nodes, we propose a simple crosslayer protocol. We show through simulation that our poweraware protocol outperforms those with no knowledge of energy.
\end{abstract}

Keywords-Social Hierarchy; Adaptive Routing; Low power

\section{INTRODUCTION}

Field zoologists require detailed data of not only intraspecific (within a species) but also interspecific (across many species) interactions. This to some extent has been addressed by the ARTS project which uses radio-tracking technology to locate VHF tagged animals [1]. However, a subtle drawback of VHF transmitters is that by design, they can be tracked from great distances - thus poachers could use the same technology to hunt animals. By using low-power transceivers on tags, a dynamic wireless network can be formed opportunistically when two or more animals are within radio range of one another. Data is then transferred in multihop fashion through the network to eventually reach exit points (base-stations). As low power links are used, and only for a short duration, tracking animals by locating the signals they emit becomes more difficult for poachers and device lifetime becomes greater. The focus of this research is a simple rule based approach to adaptive routing and medium access inspired by the self-organizing social structures that occur in nature.

Due to the limited radio range and large geographical area, a very sparse connectivity graph is formed. Connections between nodes are the exception rather than the norm, with the implication that traditional routing protocols designed for mobile ad hoc networks such as DSR [2] or AODV [3] are unsuitable as end-to-end connections are rare. The only suitable method of data delivery are epidemic type protocols [4]. These essentially are delayed controlled floods effected by store-and-forward mechanisms. However, in epidemic routing every node is involved in the flooding process, with the result that it does not scale well to large numbers of nodes without excessive energy usage and network congestion.

In a wireless network designed for animal tracking and telemetry, energy is of paramount importance, due to the logistics, cost and danger involved in sedating an animal to equip it with a collar. For this reason, a collar must last as long as possible. However, not all collars need be made equal due to the large amount of diversity presented by the carrying capacity of the animal hosts in terms of weight. Thus, different species can be equipped with collars with varying amount of battery power.

Inspired by a successful biological principle, the social dominance hierarchy, nodes form an analogous ranking based on energy. We introduce the concepts of levels within the social hierarchy and show how this results in a controllable traffic density, regardless of the number of nodes within the network. We further show how the social hierarchy can be adaptively constructed using simple rules. The social hierarchy reacts rapidly to node insertions and removals through continual node tournaments for dominance based on energy. This protocol is simple to implement in resource constrained microcontrollers, requires little in the way of storage space and scales easily to large numbers of nodes without excessive overhead.

\section{BACKGROUND}

\section{A. Related Work}

Epidemic routing is based on mimicking the spread of a disease through a population and is essentially a flooding mechanism for disconnected networks, but leads to high network resource usage [4]. To control network usage, variants have been presented in the literature, such as Spray and Wait [5] and Oracle based schemes [6]. However, none have considered the problem of heterogeneity with respect to energy. 
ZebraNet provided the first comprehensive examination of the use of wireless sensor networks for animal tracking [7], [8]. GPS equipped collars were fitted on zebras and exchange information in an epidemic fashion. Their routing algorithm is very simple and leads to buffer overflow as every node in the network stores information from every other node. They only considered fitting the collars on a single type of animal. The Shared Wireless Infostation Model (SWIM) is a routing protocol that addresses some of the issues faced by the Epidemic routing protocol [9]. Their main contribution is in the form of 'anti-packets' - messages that prevent nodes from buffering data that has already been delivered to the basestation. However, like ZebraNet, they concern themselves with instrumenting a single species - whales. Sikka et al. present a wireless sensor network designed to monitor a typical farm environment [10]. They also do not consider using the capabilities of different animals to lead to a better performing network.

\section{B. Naturally Occurring Social Hierarchies}

Social hierarchies naturally occur in a number of species and are typically motivated by differences in physical attributes such as size or weight. Some individuals can be regarded as being 'fitter' than others based upon a set of measurable characteristics. Anemones for example, form a hierarchy based upon size, in which larger anemones are more aggressive towards smaller anemones [11]. Crayfish form a social dominance structure based on length, in which the shorter crayfish defer to the largest, super-dominant individual [12]. Social hierarchies can also be found in fish (Malawi Cichlids [13] and salmon [14]); insects (ants [15], bees [16] and wasps [17]) and mammals (baboons [18] and coyotes [19]). Thus it can be seen that social hierarchies are a common organizational structure in a wide variety of organisms.

A linear social dominance hierarchy is characterized by a group that is led by the largest or fittest member - the superdominant or alpha individual. All other members of the group submit to this animal. The next in the hierarchy, the beta member is superior to all other members barring the alpha and so on. Thus, the weakest (omega) member in the pack will be subordinate to all other members. However, it must be noted that this is not a static structure, and the hierarchy adapts rapidly to changes. For example, if the alpha member becomes sick or injured, a fitter member will 'challenge' for the role of pack leader. Using these biological lessons, it can be seen how diversity in terms of fitness, however so perceived, leads to a unified, self-organizing structure. By applying these principles to the structure of a wireless network that is diverse over some attributes, a similar self-organizing hierarchy can be formed.

\section{Node diversity introduced by host carrying capability}

Animals are different with respect to their bodyweight. The size of a package that can be affixed to an animal has to be carefully considered so as to not adversely affect the host creature. A typical guideline is that a tag's weight may not exceed $5 \%$ of the body mass of the tagged animal [20]. Take for example the weight of a bull African Elephant which, when fully grown, can weigh $6000 \mathrm{~kg}$. In comparison, a small animal such as a Vervet monkey only weighs a few kilograms. Thus, for this rather restrictive example, there is a three order of magnitude difference in weight and correspondingly for the tag weight that each animal can carry. We argue that this difference should be exploited to the benefit of the operation of the network. In this way, the lightweight animals can use the capabilities of the heavyweight animals to result in a more efficient and longer lived network.

\section{A Social Fitness Hierarchy Based Upon Node ENERGY}

As some nodes have more energy than other nodes by virtue of the weight of the host, a node's role within the network should be assigned according to the amount of energy it possesses relative to its peers. Hence a node with a large amount of energy should assume a high position in a social hierarchy, and conversely a node with a small amount of energy should have a low status.

Under the assumption that the generation of messages is constant across all nodes in the network (which is realistic as there is no reason why there should be more data from a larger animal than a smaller one), the only difference in their energy use is in the communication they undertake in the form of receiving and sending messages from and to their peers respectively. Clearly then a node with a high social standing (and hence a large amount of energy) should be more active in networking tasks such as routing and forwarding messages than a low ranked node. Furthermore, a node with a large amount of energy is less likely to expire than a low energy node before it can deliver its messages, and thus should be regarded as a more attractive carrier.

First we introduce some important concepts that are used to shape traffic density through the network. Nodes have a 'rank', which is a continuous variable over the range $[0 ; 1]$ which indicates their status in the hierarchy. The rank is then quantised to form $\mathrm{N}$ discrete 'levels'. We will show how these levels control network usage.

First though, we specify our routing rule:

If node( $i$ ) and node(j) meet where Level $(j)>\operatorname{Level}(i)$ then node $(i)$ sends its buffered data to node $(j)$

As mentioned above, the position of a node within the hierarchy dictates its energy usage. This will now be shown analytically. For simplicity, the case of nodes executing a random walk over a unit toroid is considered. Although this is not entirely realistic, it does provide guidelines for the distribution of traffic, and consequently energy, with nodal level.

Consider a node at the lowest level in the structure - from the routing rule, it is clear that it will not receive messages 
from any other node in the network and it will transmit its generated messages to any higher rated node that it encounters. Thus if we define the rate of message generation to be $\lambda$ messages per unit time and the traffic density at level $\mathrm{k}$ to be $D_{k}$, then the lowest level node will have traffic density $D_{1}=\lambda$.

We define the base-station(s) to have a level of $D_{N}$ as all traffic must eventually reach the exit point from the network. Now if there are $\mathrm{N}$ levels in the hierarchy, then the second level node will generate $\lambda$ packets of its own, and also be responsible for forwarding a fraction of the first level's traffic. As nodes are executing a random walk, there will be an equal probability of meeting any higher ranked node. There are $\mathrm{N}-1$ higher energy levels in the network than Level 1 , and thus each will on average receive $\lambda(N-1)$ messages from the lowest level node. Thus, the amount of traffic that a second level node is responsible for will be:

$$
D_{2}=\lambda+\frac{\lambda}{N-1}=\lambda\left(1+\frac{1}{N-1}\right)
$$

In general, the traffic at level $\mathrm{k}$ can be expressed recursively as

$$
D_{k}=D_{k-1}\left(1+\frac{1}{N-k+1}\right)
$$

which simplifies to

$$
D_{k}=\frac{\lambda N}{N-k+1}
$$

Thus the traffic at each node grows in proportion to its level. The base-station will obviously receive all messages generated and hence will have a traffic density of $\lambda N$. This means that the majority of nodes will have very low traffic, but a few nodes will have a dramatically larger amount of traffic.

Clearly controlling the number of levels in the hierarchy (which does not need to be equal to the number of nodes in the network, as more than one node can occupy the same level in the hierarchy) affects the distribution of traffic, and hence, required node energy. For example, if there are 100 levels in the hierarchy, the highest rated non-base-station node will be have to transmit 50 times as much traffic (and hence have 50 times as much energy) as the lowest level node, whereas for an eight level structure, the highest level node will only have to transmit four times as much traffic as a low level node. Note that as long as there are equal numbers of nodes in each level of the hierarchy, the only parameter affecting traffic distribution is the number of levels, N. In this way, this protocol scales well to large numbers of nodes and traffic is predictable and controllable regardless of the number of nodes in the network. Thus, the number of levels can be chosen based on the level of diversity presented by the application.

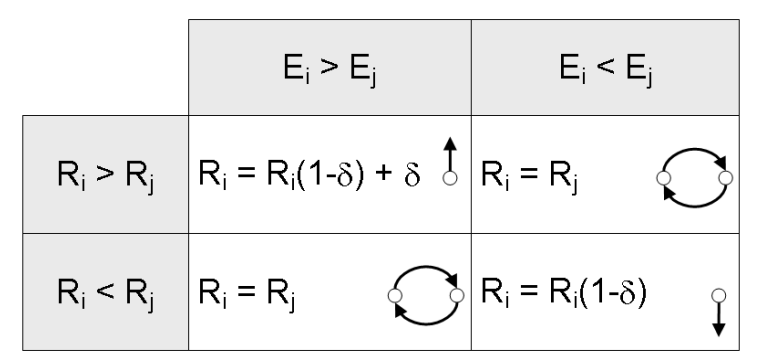

Figure 1: Tournament outcomes based on node energy (E) and perceived rankings (R). Arrows indicate how the ranking of each node changes. If a node's perception of rank is correct, then its rank is reinforced either in a positive or negative direction. If a node's energy relative to its competitor contradicts its perception of rank, then it switches its rank with that of the competitor.

This provides a design guideline for equipping nodes with different capacity batteries.

\section{DYNamically DETERMINING STATUS IN HIERARCHY}

A node's fitness can be statically set at design-time, but this will lead to a fixed hierarchy that might not actually reflect the true fitness levels of nodes. For example a highly ranked node could, as the result of a battery problem, only have a small amount of energy available. A means is needed of dynamically updating and adjusting the hierarchy much as occurs in a naturally occurring societal grouping. The hierarchy could be determined by each node periodically flooding the network with its current energy, but this does not scale well to large numbers of nodes. Instead we propose a novel method whereby nodes use locally acquired information to estimate their position in the hierarchy through a series of tournaments, in the same way animals engage in aggressive behavior in order to enforce dominance. This information is used to decide whether to stay at the current level or to move up or down the hierarchy.

When a node is first placed in the network, it has zero knowledge about the energy of its peers - thus it does not know whether it is near the top of the energy hierarchy or whether it is a low energy node. It randomly chooses a rank between 0 and 1 . When two nodes meet, they trade two pieces of information with one another - their measured energy (E), and their perceived rank $(\mathrm{R})$ within the network. Based on the information the node receives from the other node, it determines whether this newly acquired information reinforces its perception of rank or whether this contradicts its belief. There are four outcomes from the tournament - two are reinforcing (either in a positive direction or a negative direction), and the other two deal with contradiction by switching ranks. The tournament 'rules' are shown in Fig. 1. If nodes have equal energy, then they exit the tournament without altering their rank. Each node is subject to the same tournament rules, and thus based on the other node's rank and energy, each node re-assesses its position without any further communication. The nodes quantize their rank in order to determine their level within the hierarchy.

The 'switching' mechanism of exchanging rank results in 


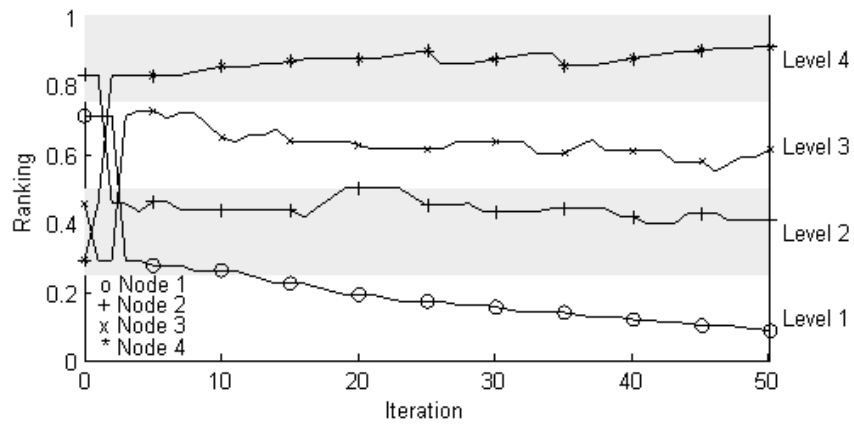

Figure 2: Rank trajectories with meeting in a four-node four-level network $(\delta=0.1)$

incorrectly ranked nodes rapidly moving through the hierarchy towards a more suitable rank. The double reinforcement strategy ensures that the hierarchy is constantly and dynamically restructured, with the alpha dominant node eventually ending up with a rank of 1 and the omega submissive node tending towards a rank of 0 . The adaption parameter $\delta(0 \leq \delta \leq 1)$ controls how dynamic the social hierarchy is. If $\delta$ is chosen too large, nodes migrate between levels too rapidly, leading to a relatively unstable social hierarchy. However, if $\delta$ is too small, the only ranks available to the nodes will be those chosen at random upon node entry into the network.

Fig. 2 demonstrates how nodes change their rank based on meetings. For each iteration, a random pair of nodes meet and exchange energy and rank information and execute the tournament rules specified in Fig. 1. It can be seen that in the beginning, nodes rapidly alter position in the hierarchy from their randomly chosen ranks. However, once the relative ranking is correct, changes to rank occur much more slowly, implying the hierarchy is stable.

\section{Results}

The performance of this scheme is assessed with respect to two multi-copy routing schemes - epidemic routing [4] and the Spray and Wait [5] protocol and a single-copy greedy randomized protocol with probability of handover of 1 . The simulation environment is a square of side $10 \mathrm{~km}$, radio range is circular of $500 \mathrm{~m}$ and nodes move according to the random waypoint mobility model with a non-zero minimum speed (after [21]) and maximum speed of $5 \mathrm{~m} / \mathrm{s}$. Nodes randomly generate information with a Poisson rate of 1 packet every 100 seconds. We assume that transmission of a single packet consumes 1 unit of energy, and the overhead of communicating rank and energy for our protocol uses 0.05 units of energy. Nodes are assigned energies in proportion to the traffic densities calculated using Eq. (3) - the lowest level nodes have an energy of 1000 units and the highest level nodes have an energy of 10000 units. We use a 10 level network in this simulation.

The results from the simulation are shown in Fig. 3 demonstrating the mean time to first node expiry. As expected, epidemic routing performs the worst, exhausting nodes rapidly through excessive traffic usage. Spray and Wait

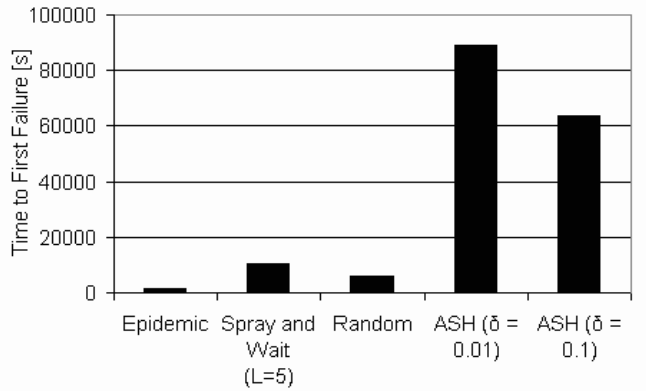

Figure 3: Mean time to first node expiry for the various protocols $(\mathrm{ASH}=$ Adaptive Social Hierarchy)

achieves a reasonable performance through controlling the number of packets transmitted by the scheme and is better than the randomized handover algorithm. However, the power of our protocol is clear - by making the protocol energy aware, low energy nodes participate less in network activity, resulting in a greater overall lifetime. Two different values of the adaption parameter, $\delta$, are used. The value of $\delta=0.1$ leads to more rapid node exhaustion as the social hierarchy is less stable than $\delta=0.01$ resulting in nodes being incorrectly ranked.

\section{Cross Layer Protocol}

Our scheme lends itself well to a so-called cross-layer routing protocol - one which uses the same information at multiple levels within the protocol stack. These are generally treated with caution, as they can lead to unintended feedback cycles [22]. However, ours is extremely simple and arises from the fact that information only flows up the hierarchy. Thus, if medium access is controlled according to hierarchical level, we can further conserve energy of the low energy nodes. This is because they will not have to compete with higher ranked nodes for access to the medium.

A diagram of the slotted access scheme for a 5 level hierarchy is shown in Fig. 4. There are 4 active slots in each epoch, which are separated by an Inter-Epoch Slot (IES) where all the nodes are asleep. The level 5 node is a basestation and thus will spend all of the active slots awake and listening for transmissions from low level nodes. It can be seen that while this scheme leads to greater energy consumption for highly rated nodes, low level nodes get preferential access to the medium, thus boosting their lifetime. As levels in the hierarchy are not statically assigned, if a node assumes a role that depletes its energy rapidly relative to its peers, its relative fitness will decrease and it will descend the hierarchy. In doing so, it will consume less energy. Thus a form of local negative feedback is created by this action.

\section{Future DiRECTIONS}

The routing protocol here mainly concentrates on a sole element of diversity between nodes, namely energy. However, the Animal Kingdom is a rich source of diversity with many facets. We are currently investigating a more advanced hierarchy which exploits correlations in time and 


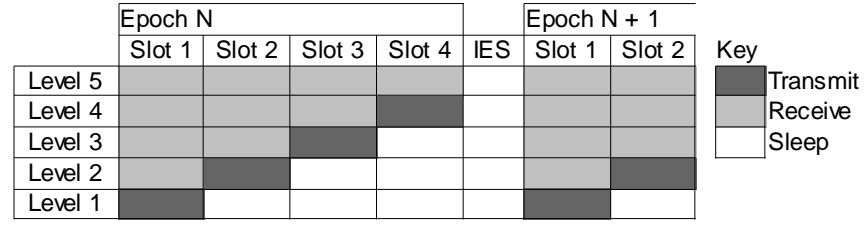

Figure 4: Cross layer slotted access scheme. Slots are assigned based on node level. Low level nodes spend the majority of their time sleeping which conserves their energy.

space, as would be expected with real animal motion. As it stands, our protocol is a single-copy routing strategy, but it would be simple to modify the routing rules such that there is increasing replication of messages as a function of hierarchical level. In this way, delivery would be direct at the low levels of the hierarchy and close to epidemic at the higher levels, leading to an element of redundancy.

\section{ConcLusion}

This research takes a very common (and hence successful) structure in the Animal Kingdom, the social hierarchy, and adapts it to a wireless sensor network designed for diverse animal monitoring and tracking. The social hierarchy is thought to reduce conflict in animal groups, and here it is used to reduce energy use for low ranked nodes. Based on a simple routing rule, and a means of dynamically assessing global energy distribution through locally acquired information, nodes adaptively choose an activity level that dictates their role within the hierarchy. Each node chooses its role itself, with no centralized control, resulting in a system that scales well to large numbers of nodes. This work is a novel application of a common ethological structure that results in a powerful routing algorithm .

\section{ACKNOWLEDGMENTS}

The authors would like to thank Professor Braae and the anonymous reviewers for their helpful comments. Andrew Markham is grateful to UCT for funding assistance.

\section{REFERENCES}

[1] C. Carroll. Ocelots: Following the Stealth Hunter. National Geographic, 66-77, November 2005.

[2] D. B. Johnson, D. A. Maltz and J. Broch. DSR: the dynamic source routing protocol for multi-hop wireless ad hoc networks. In Ad hoc networking, Addison-Wesley, 2001.

[3] C. E. Perkins, E. M. Belding-Royer and S. R. Das. Ad-hoc on demand distance vector routing. IETF MANET DRAFT, 2002.
[4] A. Vahdat and D. Becker. Epidemic Routing for Partially-Connected Ad Hoc Networks. Duke Tech Report CS-2000-06, 2000.

[5] T. Spyropoulos, K. Psounis, and C. Raghavendra. Spray and wait: An efficient routing scheme for intermittently connected mobile networks, In Proceedings WDTN, 2005

[6] S. Jain, K. Fall, R. Patra. Routing in a Delay Tolerant Network. In Proceedings ACM SIGCOMM, Portland, Oregon (2004)

[7] P. Juang, H. Oki, Y. Wang, M. Martonosi, L. Peh and D. Rubenstein. Energy-Efficient Computing for Wildlife Tracking: Design Tradeoffs and Early Experiences with ZebraNet. ASPLOS X, San Jose, CA, USA, 2002

[8] P. Zhang, C. M. Sadler, S. A. Lyon and M. Martonosi. Hardware Design Experiences in ZebraNet. SenSys, Baltimore, Maryland, USA, 2004

[9] T. Small, Z. J. Haas, A. Purgue and K. Fristrup (2003): The Shared Wireless Infostation Model: A New Ad Hoc Networking Paradigm (or Where There is a Whale, There is a Way). Fourth ACM International Symposium on Mobile AdHoc Networking \& Computing, 2003

[10] P. Sikka, P. Corke, P. Valencia, C. Crossman, D. Swain and G. BishopHurley. Wireless adhoc sensor and actuator networks on the farm. In Proceedings of the Fifth international Conference on Information Processing in Sensor Networks, Nashville, Tennessee, USA, April 2006

[11] R. C. Brace and J. Pavey. Size-dependent dominance hierarchy in the anemone Actinia equina. Nature, 273:752-753, 1978

[12] F. A. Issa, D. J. Adamson and D. H. Edwards. Dominance hierarchy formation in juvenile crayfish procambarus clarkii. Journal of Experimental Biology, 202(24):3497-3506, 1999

[13] I. D. Chase, C. Tovey, D. Spangler-Martin and M. Manfredonia. Individual differences versus social dynamics in the formation of animal dominance hierarchies. PNAS, 99(8):5744-5749, 2002

[14] S. Nakano. Variation in agonistic encounters in a dominance hierarchy of freely interacting red-spotted masu salmon (Oncorhynchus masouishikawai). Ecology of Freshwater Fish, 3(4):153-158, 1994

[15] T. Monnin and C. Peeters. Dominance hierarchy and reproductive conflicts among subordinates in a monogynous queenless ant. Behavioral Ecology, 10(3):323-332, 1999

[16] B. H. Smith. Effects of genealogical relationship and colony age on the dominance hierarchy in the primitively eusocial bee Lasioglossum zephyrum. Animal behaviour, 35(1):211-217, 1987

[17] R. H. Barth, L. J. Lester, P. Sroka, T. Kessler and R. Hearn. Juvenile hormone promotes dominance behavior and ovarian development in social wasps (Polistes annularis). Cellular and Molecular Life Sciences, 31(6):691 - 692, 1975

[18] G. Hausfater. Dominance and reproduction in Baboons (Papio cynocephalus). Contrib. Primatology, 7:1-150, 1975

[19] E. M. Gese, R. L. Ruff and R. L. Crabtree. Foraging ecology of coyotes (Canis latrans): The influence of extrinsic factors and a dominance hierarchy. Canadian Journal of Zoology, 74(5):769-783, 1995

[20] W. Cochran. Wildlife Telemetry. In S. D. Schemnitz (Ed.), Wildlife management techniques manual, 1980

[21] J. Yoon, M. Liu and B. Noble. Random way point considered harmful. In Proceedings of IEEE INFOCOM, San Fransisco, California, USA, April 2003

[22] V. Kawadia and P. R. Kumar. A cautionary perspective on cross layer design. IEEE Wireless Communications Magazine, February 2005 